\title{
Silicon Raman laser, amplifier, and wavelength converter
}

Bahram Jalali, Ozdal Boyraz, Dimitri Dimitropoulos, Varun Raghunathan

Bahram Jalali, Ozdal Boyraz, Dimitri Dimitropoulos, Varun Raghunathan, "Silicon Raman laser, amplifier, and wavelength converter," Proc. SPIE 5840, Photonic Materials, Devices, and Applications, (7 July 2005); doi: 10.1117/12.608446

SPIE. Event: Microtechnologies for the New Millennium 2005, 2005, Sevilla, Spain 


\title{
Silicon Raman laser, amplifier, and wavelength converter
}

\author{
Bahram Jalali, Ozdal Boyraz, Dimitri Dimitropoulos, Varun Raghunathan \\ University of California, Los Angeles \\ Los Angeles, CA 90095-1594 \\ jalali@ucla.edu
}

\section{Introduction}

Silicon is the "bread and butter" of the electronics industry. Its combination of technological sophistication and economics of scale of is unparalled in the history of the industrial age. Ideally, photonic devices should also be manufactured in silicon. However, with the exception of one or two devices that are in low-volume production [1] the silicon photonics industry is virtually non existent. Technologically, this can be attributed to the premature perception that physical properties of silicon do not lend themselves to important functions such as light emission, amplification and wavelength conversion. Yet, photonic devices are essential building blocks of fiber optic networks that form the backbone of the internet. Being able to tap into silicon's vast manufacturing base will reduce the cost of photonic devices which in turn will accelerate penetration of optics into the access portion of the internet. Silicon photonics can thus be viewed as a key to broadband access for the masses. Additionally, the technology can solve important problems in today's computing systems as well as spawn new industries of its own. For example, as the trend to reducing device dimensions continues, a significant bottleneck has appeared at the electronics interconnect level, where a large gap exists between individual device speeds and the speed of interconnects that link them [2,3]. Optical interconnects can potentially solve this important problem.

The compatibility of silicon photonics with silicon IC manufacturing, and separately with silicon MEMS technology, has generated significant attention to this field. In 2004, the U.S. Defense Advanced Research Project Agency (DARPA) launched the Electronic and Photonic Integrated Circuit (EPIC) program, the first of its kind dedicated to silicon photonics. The four year program is funding research and development at universities and industry with the ultimate goal of producing production capable silicon optoelectronic circuits. The first International Conference on Group IV Photonics was also launched in 2004. The year was also witness to the demonstration of the first silicon laser [4]. The rapid pace of progress is continuing and the first quarter of 2005 has already seen the demonstration of direct electrical modulation of the Raman laser [5] and report of the first CW silicon Raman laser [6].

Compared to other integrated optics platforms, a distinguishing property of silicon is the tight optical confinement made possible by the large index mismatch between the silicon and $\mathrm{SiO}_{2}$. While a myriad of high performance passive devices were demonstrated in the 1990's [7], creation of active devices proved to be much more difficult. Unfavorable physical properties such as the near-absence of Pockel's effect caused by the symmetric crystal structure, and the lack of efficient optical transitions, due to the indirect band structure, were the culprits.

Raman scattering was proposed and demonstrated in 2002 as a mean to bypass these limitations and to create optical amplifiers and lasers in silicon [8]. The approach was motivated by the fact that the stimulated Raman gain coefficient in silicon is $10^{3}-10^{4}$ times larger than that in fiber. The modal area in a silicon waveguide is roughly 100 times smaller than in fiber resulting in a proportional increase in optical intensity. The combination makes it possible to realize chipscale Raman devices that normally require kilometers of fiber to operate. The initial demonstration of spontaneous Raman emission from silicon waveguides in 2002 was followed by the first demonstration of stimulated Raman scattering [9] and parametric Raman wavelength conversion [10] both in 2003. Other merits of Raman effect include the fact that it occurs in pure silicon and hence does not require rare earth dopants (such as Erbium), and that the spectrum is widely tunable through the pump laser wavelength. 


\section{Raman Amplification in Silicon}

Classical electrodynamics provides a simple and intuitive macroscopic description of the Raman scattering process. In the spontaneous scattering, thermal vibrations of lattice at frequency $\omega_{\mathrm{v}}(15.6 \mathrm{THz}$ in silicon) produce a sinusoidal modulation of the susceptibility. The incident pump field induces an electric polarization that is given by the product of the susceptibility and the incident field. The beating of the incident field oscillation $\left(\omega_{\mathrm{p}}\right)$ with oscillation of the susceptibility $\left(\omega_{v}\right)$ produces induced polarizations at the sum frequency, $\omega_{\mathrm{p}}+\omega_{\mathrm{v}}$, and at the difference frequency $\omega_{\mathrm{p}}-\omega_{\mathrm{v}}$. The radiation produced by these two polarization components is referred to as anti-Stokes and Stokes waves, respectively. Quantum statistics dictates that the ratio of Stokes power to anti-Stokes power is given by $\frac{1+N}{N}$, where $N=\left[\exp \left(\hbar \omega_{v} / k T\right)-1\right]^{-1}$ is the Bose occupancy factor and has a value of $\sim 0.1$ for silicon at room temperature.

The same model can be extended to describe Stimulated Raman scattering [11]. Here, we assume the pump field and the Stokes field are present, with a frequency difference equal to the atomic vibrational frequency. The latter can be due to spontaneous emission, or in the case of a Raman amplifier, it is the input signal that is to be amplified. The two fields (pump and Stokes) create a force that stimulates atomic vibrations, even in the absence of a dipole moment. This can be understood as follows. If $E$ is the total field comprising of pump and Stokes, and $\chi$ is the susceptibility, the energy stored in the field, $V=\frac{(1+\chi)}{2} E \cdot E^{*}$, will have a component oscillating at $\omega_{\mathrm{p}}-\omega_{\mathrm{s}}=\omega_{\mathrm{v}}$. Through the modulation of the susceptibility with displacement, $Q$, this will produce a force $F \propto \frac{\partial V}{\partial Q} \approx \frac{\partial \chi}{\partial Q} E_{p} \cdot E_{s} \cdot \exp \left(-\omega_{v} \cdot t\right)$. This deriving force will enhance atomic oscillations which in turn will increase the amplitude of the Stokes field, $E_{s}$. This positive feedback phenomenon is called stimulated Raman scattering and results in the amplification of the Stokes field.

While providing an intuitively appealing description of Raman scattering, the macroscopic model described above does not account for detailed processes responsible for Raman scattering in silicon. The microscopic picture reveals that the direct coupling of light with atomic vibrations, described by the interaction Hamiltonian involving photons and phonons, is very weak. This is generally true in semiconductors owing to the large atomic mass that appears (squared) in the denominator of the cross section. In silicon, the lack of lattice polarization further underscores this fact. Electrons mediate the Raman scattering process in silicon. Microscopically, the scattering proceeds in three steps [12]. In step one, the incident photon excites the semiconductor into an intermediate step by creating an electron-hole pair. In step two, the pair is scattered into another state by emitting a phonon via the electron-phonon interaction Hamiltonian. In step three, the electron-hole pair in the intermediate step recombines radiatively with emission of a scattered photon. While electrons mediate the process, they remain unchanged after the process. Furthermore, transitions involving electrons are virtual and hence do not have to conserve energy although momentum must be conserved.

Raman describes the scattering process involving the optical phonon branches of atomic vibrations (as opposed to Brillouin which describes scattering involving acoustic phonons). In first order scattering, only one phonon is involved and momentum conservation implies that only zone-center phonons can participate. Higher order Raman scattering involves multiple phonons which can be from any point in the Brillouin zone as long as their total momentum equals the (negligible) photon momentum. In silicon, the zone center optical phonon is triply degenerate with a frequency of $15.6 \mathrm{THz}$. Figure 1 shows the typical Raman spectrum of silicon with the pump-Stokes separation of $15.6 \mathrm{THz}$ highlighted. The

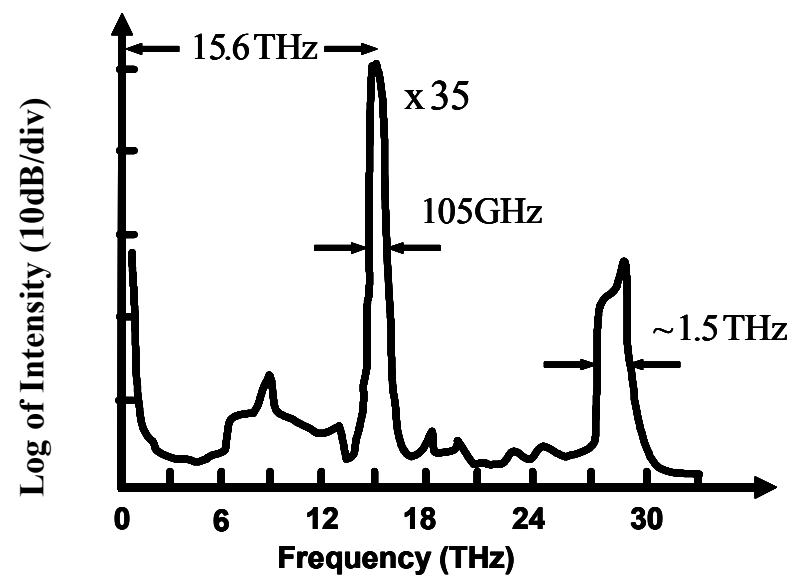

Figure 1. Spontaneous Raman spectra of silicon showing $1^{\text {st }}$ and $2^{\text {nd }}$ order Stokes emission. Frequency is plotted relative to pump frequency. Anti-Stokes spectrum is not shown. 
first order resonance, which is of primary importance here, has a FWHM of approximately 100GHz [13]. This imposes a maximum information bandwidth of approximately $100 \mathrm{GHz}$ that can be amplified. The Raman linewidth becomes broader when a broadband pump is used.

Crystal symmetry imposes a selection rule that dictates which scattering geometries are allowed. The spontaneous scattering efficiency, $S$, is given by,

$$
S=S_{0} \sum_{k=1,2,3}\left|\hat{e}_{s} \cdot R_{k} \cdot \hat{e}_{p}\right|^{2} .
$$

Unit vectors $\hat{e}_{p}$ and $\hat{e}_{s}$ denote the polarization of the pump and Stokes electromagnetic fields. $S_{0}$ contains intrinsic microscopic property of silicon including derivatives of the polarizability, and the absolute amplitude of the displacement of the zone-center optical phonons. The sum runs over the three Raman matrices, each corresponding to the phonon displacement along one of the three principle axis of the crystal [14]:

$$
\vec{R}_{1}=\left[\begin{array}{lll}
0 & 1 & 0 \\
1 & 0 & 0 \\
0 & 0 & 0
\end{array}\right], \vec{R}_{2}=\left[\begin{array}{lll}
0 & 0 & 1 \\
0 & 0 & 0 \\
1 & 0 & 0
\end{array}\right], \vec{R}_{3}=\left[\begin{array}{lll}
0 & 0 & 0 \\
0 & 0 & 1 \\
0 & 1 & 0
\end{array}\right]
$$

Table 1 below shows the relative spontaneous Raman intensities obtained for different scattering configurations in silicon. In the Table, the vectors $\hat{k}_{p}$ and $\hat{k}_{s}$ denote the pump and Stokes propagation directions, respectively.

\begin{tabular}{|c|c|c|c|}
\hline$\hat{k}_{t} \& \hat{k}_{s}$ & $\hat{e}_{t}$ & $\hat{e}_{s}$ & $\begin{array}{c}\text { Relative } \\
\text { Efficiency }\end{array}$ \\
\hline$[1 \overline{1} 0]$ & {$[110]$} & {$[110]$} & $S_{O}$ \\
\hline$[1 \overline{1} 0]$ & {$[110]$} & {$[001]$} & $S_{O}$ \\
\hline$[1 \overline{1}]$ & {$[111]$} & {$[111]$} & $4 S_{O} / 3$ \\
\hline$[111]$ & {$[1 \overline{1} 0]$} & {$[1 \overline{2}]$} & $2 S_{O} / 3$ \\
\hline$[111]$ & {$[11 \overline{2}]$} & {$[11 \overline{2}]$} & $S_{O}$ \\
\hline$[0 \overline{1} 1]$ & {$[011]$} & {$[100]$} & $S_{O}$ \\
\hline$[0 \overline{1} 1]$ & {$[011]$} & {$[011]$} & $S_{O}$ \\
\hline
\end{tabular}

\section{Table 1: Spontaneous Scattering efficiency for various wave vector and polarization direction of Pump and Stokes fields}

The Raman gain coefficient, $\mathrm{g}_{\mathrm{R}}$, can be obtained from the spontaneous efficiency, $\mathrm{S}$, using the Einstein relation, as [15]: 


$$
g_{R}=\frac{8 \pi c^{2} \omega_{p}}{\hbar \omega_{s}^{4} n^{2}\left(\omega_{s}\right)(N+1) \Delta \omega} S
$$

Substituting the appropriate values, the gain coefficient is obtained as $\sim 76 \mathrm{~cm} / \mathrm{GW}[8]$. This uses $S=8.4 \times 10^{-7} \mathrm{~cm}^{-1} \mathrm{Sr}^{-1}$ which was obtained by extrapolating the values measured $1.1 \mu \mathrm{m}$ wavelength to 1.55 using a $\lambda^{-4}$ relation. This is in the same order of magnitude but several times larger than the values extracted from Raman gain measurements ( 20 $\mathrm{cm} / \mathrm{GW})$ at $1.55 \mu \mathrm{m}$, nonetheless, when compared to silica $\left(0.93 \times 10^{-2} \mathrm{~cm} / \mathrm{GW}\right)$, the Raman gain in silicon is $10^{3}-0^{4}$ times larger. Such a large difference has its origin in the much narrower linewidth of the Raman spectrum in crystalline silicon, compared to the amorphous fiber.

It is customary to describe the induced polarization for the case of stimulated Raman scattering through the nonlinear susceptibility, $\chi_{i j m n}^{R}$, defined by the following expression:

$$
P_{i}^{N L}\left(\omega_{s}\right)=\varepsilon_{0} \chi_{i j m n}^{R} E_{j}\left(\omega_{p}\right) E_{m}\left(-\omega_{p}\right) E_{n}\left(\omega_{s}\right)
$$

On the other hand, the atomic displacement can be obtained using a classical harmonic oscillator model [11] with the driving force described above. By comparing the induced polarization suggested by the displacement with the definition above, one arrives at the following expression for the induced Raman susceptibility,

$$
\chi_{i j m n}^{R}=2 \Gamma \omega_{v} \frac{2 n c g_{R}}{\omega_{s}\left(\mu_{0} / \varepsilon_{0}\right)^{1 / 2}} \frac{\sum_{k=1,2,3}\left(R_{i j}\right)_{k}\left(R_{m n}\right)_{k}}{\left(\omega_{v}^{2}-\left(\omega_{p}-\omega_{s}\right)^{2}-2 i \Gamma\left(\omega_{p}-\omega_{s}\right)\right.},
$$

Where $\Gamma$ is the dissipative term in the harmonic oscillator equation and $n$ is the refractive index. Crystal symmetry consideration, described by the Raman tensor, $R$, leads to a total of 12 equal non-vanishing components that have the indices of the form:

$$
1221=1212=2112=2121=1331=1313=3113=3131=2332=2323=3223=3232
$$

The induced susceptibility is related to the Raman gain coefficient as [14]:

$$
\chi_{1221}^{R}\left(\omega_{p}-\omega_{s}=\omega_{v}\right)=\frac{i}{\left(\mu_{0} / \varepsilon_{0}\right)^{1 / 2}} \frac{2 n c g_{R}}{\omega_{s}}=11.2 \times 10^{-14} i \frac{\mathrm{cm}^{2}}{V^{2}}
$$

Another nonlinear optical effect in semiconductors is the wo-photon-absorption (TPA). This is a deleterious effect that results in pump depletion and generation of free carriers. These carriers give rise to a broadband absorption spectrum through the free carrier plasma effect. TPA has been shown to be negligible from the point of view of pump depletion [9]. This is plausible since the TPA coefficient in silicon, $\beta$, is relatively small, $\sim 0.5 \mathrm{~cm} / \mathrm{GW}$. On the other hand, absorption by TPA-generated free carriers is a broadband process that competes with the Raman gain. The effect has been identified as a limiting factor in all-optical switching in III-V semiconductor waveguides [16-20]. It has also been discussed as a potential limit to achievable Raman gain in GaP waveguides [21], although a Raman gain of 24dB was demonstrated in these waveguides [22]. More recently, TPA-induced FCA has been measured in silicon waveguides in the context of Raman process [23,24] and in transmission of ultra short pulses in silicon waveguides [25].

The magnitude of TPA induced free carrier absorption depends on free carrier concentration through the relation: $\alpha^{\mathrm{FCA}}=$ $1.45 \times 10^{-17}(\lambda / 1.55)^{2} \cdot \Delta \mathrm{N}$, where $\lambda$ is the wavelength, in microns, and $\Delta \mathrm{N}$ is the density of electron-hole pairs [26,27]. The latter is related to the pump intensity, $I_{p}$, by 


$$
\Delta N=\beta \cdot I_{p}^{2} \cdot \tau_{\text {eff }} /(2 \cdot h v) .
$$

Where $h v$ is the pump photon energy, and, $\tau_{\text {eff }}$, is the effective recombination lifetime for free carriers. This equation neglects the contribution to free carrier generation due to pump-signal TPA and hence is valid in the regime where Stokes intensity $\left(I_{s}\right) I_{s}<<I_{p}$.

The fundamental parameter that governs the TPA induced loss, and hence the success of Raman based devices is the recombination lifetime, $\tau_{\text {eff. }}$ It is well known that the recombination lifetime in SOI is much shorter than that in a bulk silicon sample with comparable doping concentration. This lifetime reduction is due to the presence of interface states at the boundary between the top silicon and the buried oxide layer. This effect depends on the method used for preparation of the SOI wafer and the film thickness, with measured and expected values ranging between $10 \mathrm{~ns}-200 \mathrm{~ns}$ [28-30]. In SOI waveguides the lifetime is further reduced to a few nano seconds, or even below in the case of submicron

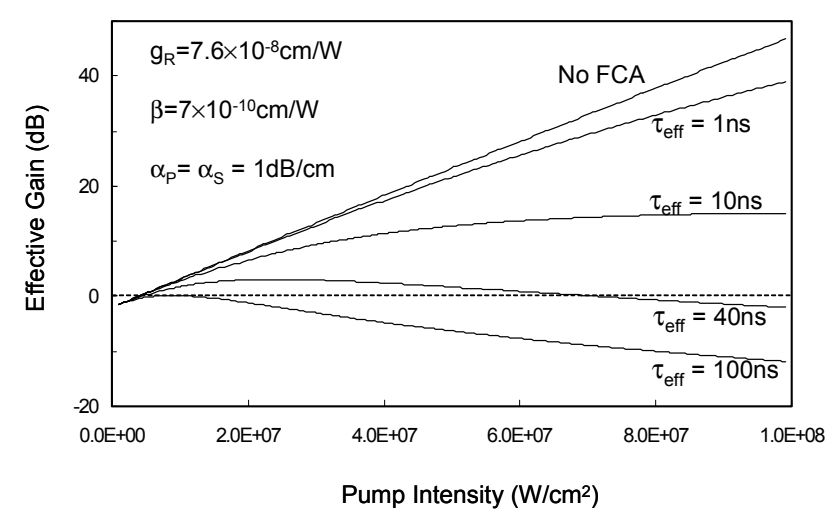

Figure 2. Impact of carrier lifetime on achievable C.W. Raman gain. Gain increases with intensity while loss rises as intensity squared and dominates when lifetime is long. waveguides, due to the recombination at the etched waveguide facets and, in the case of rib waveguides, due to diffusion into the slab regions [30]. The lifetime can be further reduced by application of a reverse bias $p$-n junction $[23,24,30]$ or by introduction of midgap states through high energy irradiation, and gold or platinum doping. Modest amount of C.W. gain has been observed in deep submicron waveguides [31] where the impact of surface and interface recombination plays a critical role in reducing the lifetime. $\mathrm{CW}$ gain has also been demonstrated by sweeping the free carriers using a reverse bias p-n junction [32]. This approach is further discussed in the context of Raman laser below.

The plot of the net Raman gain as a function of C.W. pump intensity for a waveguide of length, $L=1.9 \mathrm{~cm}$, and propagation loss $1 \mathrm{~dB} / \mathrm{cm}$, is shown in Fig. 2 [24] for different free carrier lifetime values. The plot shows that more than $10 \mathrm{~dB}$ of gain can be obtained with a pump intensity of less than $100 \mathrm{MW} / \mathrm{cm}^{2}$. Gain increases with intensity while the

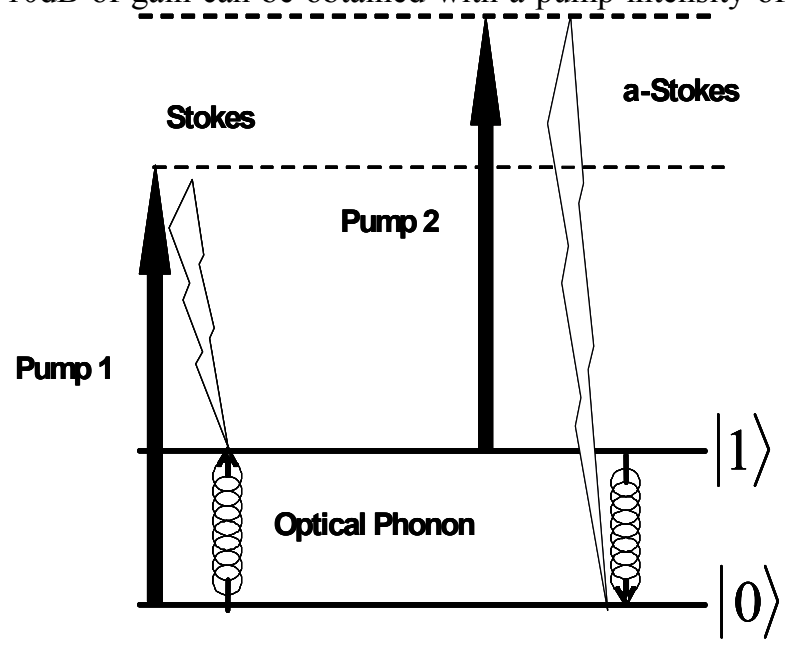

Figure 3. Energy-level representation of Raman wavelength conversion process. $\mid 1>$ and $\mid 0>$ are vibrational states and arrow represent virtual transitions. A phonon is created and annihilated leaving the phonon population unchanged. loss rises as intensity squared and dominates when lifetime is long. The pump is assumed to be a monochromatic source. The finite linewidth of the pump laser will result in a lower gain than what is predicted in Fig. 2. It is clear that to create a successful amplifier, an effective lifetime of $\leq 1 \mathrm{~ns}$ is required.

\section{Raman Wavelength Conversion}

As mentioned above, the Raman scattering spectrum also contains an anti-Stokes wave that is up shifted from the pump by the $15.6 \mathrm{THz}$ phonon frequency. The gain coefficient for the anti-Stoke wave will have a negative sign, indicating that an incident anti-Stoke wave will be attenuated. However, anti-Stoke signal can be generated through Four Wave Mixing (FWM) induced through the Raman susceptibility, in much the same way that conventional FWM takes place via the electronic $3^{\text {rd }}$ order nonlinear susceptibility (responsible for the Kerr effect). In the Raman process, the energy conservation dictates that $\omega_{a s}=2 \omega_{p}-\omega_{s}$, and momentum conservation results in the so-called phase matching condition, with the total phase mismatch defined as: 


$$
\Delta \beta=2 \beta_{p}-\beta_{s}-\beta_{a s}
$$

Where, $\beta$, is the wave-vector for the given wavelength, and the corresponding mode of polarization $\mu$ ( 1 for $\mathrm{TE}_{0}$ and 2 for $\mathrm{TM}_{0}$ ). As $\Delta \beta$ approaches zero, pump, Stokes and anti-Stokes waves experience coherent interaction. This phenomenon is referred to as Coherent anti-Stokes Raman Scattering (CARS) in the spectroscopy literature and was first observed in silicon in 2003 [10]. As shown in Figure 3, the process the creation of the anti-Stokes photon is accompanied by creation and annihilation of a zone center phonon.

The conversion efficiency is highly sensitive to the phase mismatch and, in general, the efficiency has a $\operatorname{sinc}^{2}$ dependence on phase mismatch. In silicon, phase mismatch is dominated by the material dispersion, as waveguide dispersion is relatively negligible unless in waveguides with submicron modal dimensions. In such devices, waveguide dispersion provides a mean to compensate for material dispersion. Other means of phase matching include the use of waveguide birefringence and/or strain. At phase matching, the evolution of Stokes and anti-Stokes fields, $E(z)$, along the waveguide length, $z$, is given by,

$$
\begin{aligned}
& E_{S}(z)=E_{S}(0)+\left(E_{S}(0)+E_{a S}^{*}(0)\right) g_{R} I_{P} z / 2, \\
& E_{a S}^{*}(z)=E_{a S}^{*}(0)-\left(E_{S}(0)+E_{a S}^{*}(0)\right) g_{R} I_{P} z / 2 .
\end{aligned}
$$

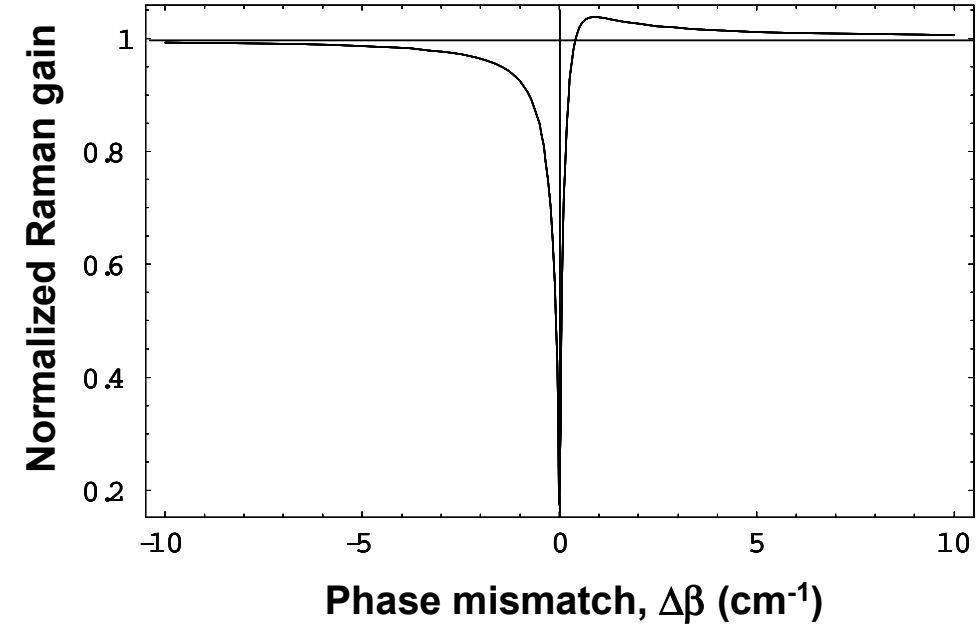

Figure 4. The variation of normalized Raman gain as a function of phase mismatch. At zero phase mismatch, Raman gain is suppress in favor of parametric Stokes to anti-Stokes conversion.

The above equation predicts a linear increase in the fields with distance which holds true for small propagation lengths. Once the Stokes and anti-Stokes fields become equal in amplitude, no further change takes place leading to a saturation effect. The characteristic length is very long, therefore, this regime is not expected to occur in chip-scale devices.

Figure 4 shows the normalized Raman gain as a function of phase mismatch. At large values of $|\Delta \beta|$, stimulated Raman amplification is the predominant effect and leads to the amplification of the Stokes signal with an effective gain given by $g_{R}$. Under phase matching condition, Raman gain is suppressed and the parametric coupling of pump and Stokes to anti-Stokes dominates. In this region, Stokes, anti-Stokes, and pump fields are strongly coupled and parametric conversion dominates. For small, positive values of $\Delta \beta$, the normalized gain slightly exceeds unity due to modulation instability. This effect has also been predicted and observed in optical fibers [33].

\section{Experimental Demonstrations}

Experimental results shown in Figure 5a highlight the competition between Raman gain and TPA induced free carrier absorption. Here a C.W. pump emitting at $1427 \mathrm{~nm}$ was used along with a tunable C.W. signal laser. The plot shows the amplification of the signal laser as it is tuned across the Raman resonance. The data clearly shows the competition between the resonant Raman amplification and the broadband pump-induced absorption. It is clear that further increase in pump intensity is futile as the intensity-squared increase of TPA-induced loss dominates over Raman gain which increases linearly with pump intensity. Also shown, in Figure 5b, is the measured spontaneous emission spectra from the 


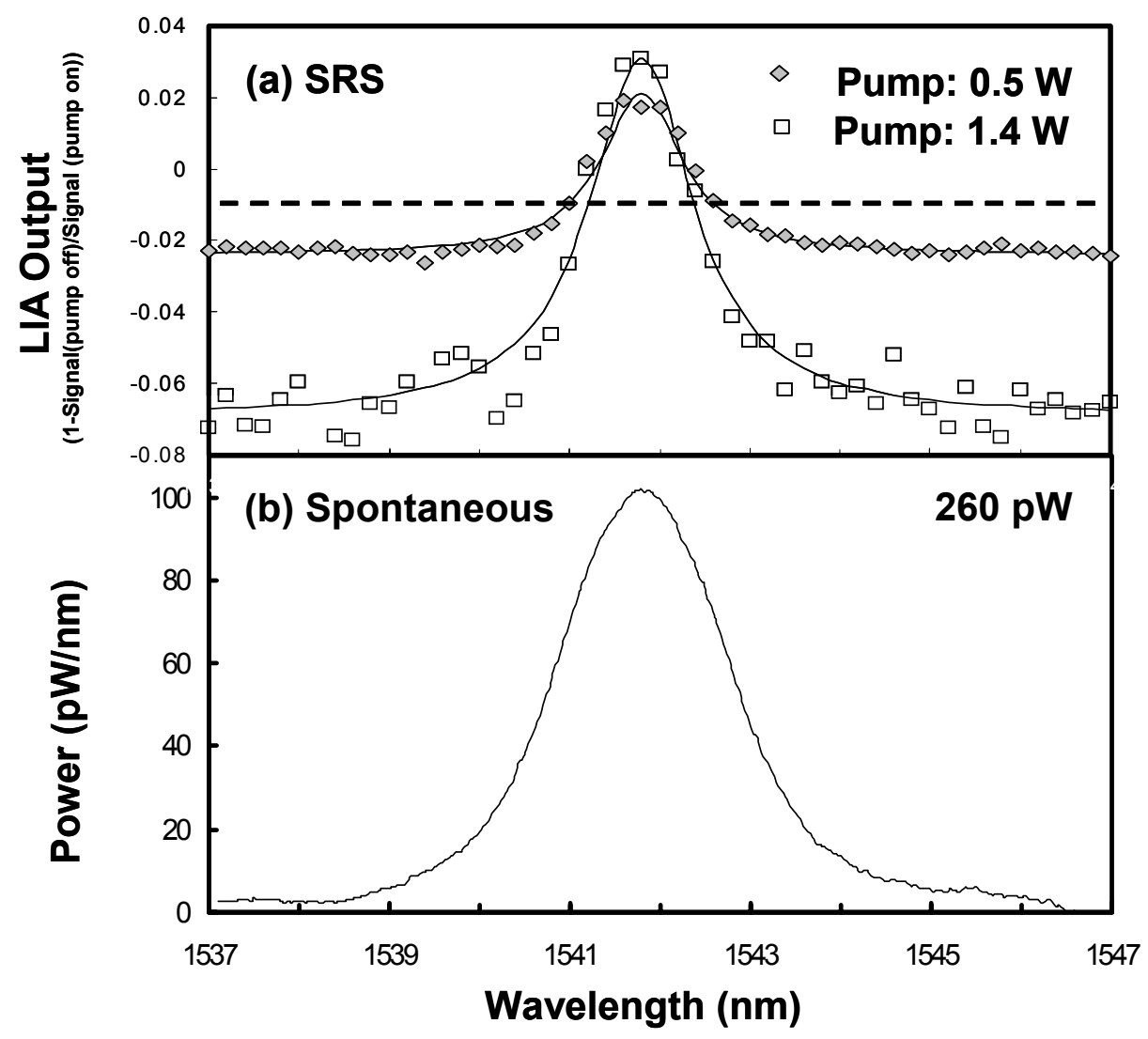

Figure 5. (a) Measured spectral characteristic of Stimulated Raman scattering (SRS) in an SOI waveguide, under CW pumping. Two different input pump powers are shown, to illustrate the effect of TPAinduced free carrier absorption. (b) measured spontaneous emission spectrum.

same waveguides. The observed FWHM is approximately twice the $100 \mathrm{GHz}$ intrinsic Raman linewidth, a feature caused

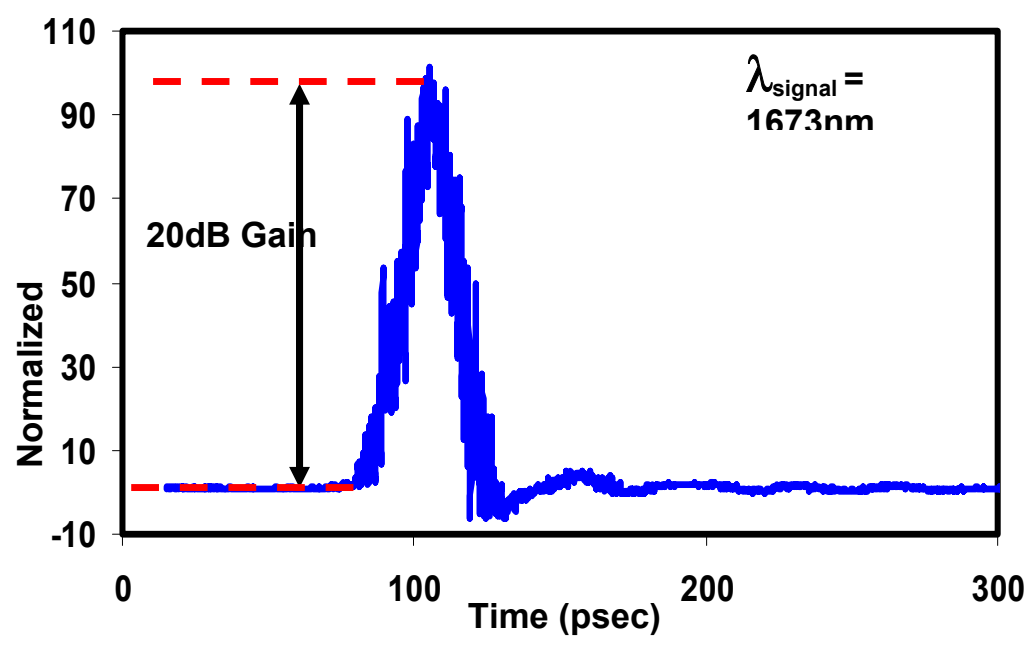

Figure 6. Time resolved Raman amplification with the signal laser at 1673nm. A pump on-off gain of $20 \mathrm{~dB}$ is obtained. Pump pulse wavelength is $1540 \mathrm{~nm}$. by the finite linewidth of the pump laser.

One method for avoiding free carrier accumulation and the concomitant loss is pulsed pumping. [34-38]. As long as the pump pulse period is longer than the free carrier lifetime the free carrier accumulation can be mostly eliminated. Figure 6 shows the measured change in $\mathrm{CW}$ signal beam (tuned to the peak of Stokes resonance) due to the pump pulse. The pump source was a modelocked fiber laser with $25 \mathrm{MHz}$ repetition rate and $\sim 1$ ps output pulse width. Since phonon response time in silicon is more than $3 \mathrm{ps}$, the pulse width is broadened by using a spool of standard single mode optical fiber. Maximum pump on-off gain of $20 \mathrm{~dB}$ has been obtained. Taking into account the losses in the waveguide, 
we obtain a net waveguide gain of $13 \mathrm{~dB}$ [38]. This gain includes waveguide propagation losses but not the fiberwaveguide coupling loss. In separate devices that have adiabatic mode tapers, we have shown net fiber-to-fiber gain of $11 \mathrm{~dB}$ under similar pulse pumping scheme [34].

The net free carrier loss and the free carrier lifetime can be measured by performing the same measurement with the signal laser tuned away from the Raman peak. This is shown in Figure 7. Maximum loss due to the combined FCA loss

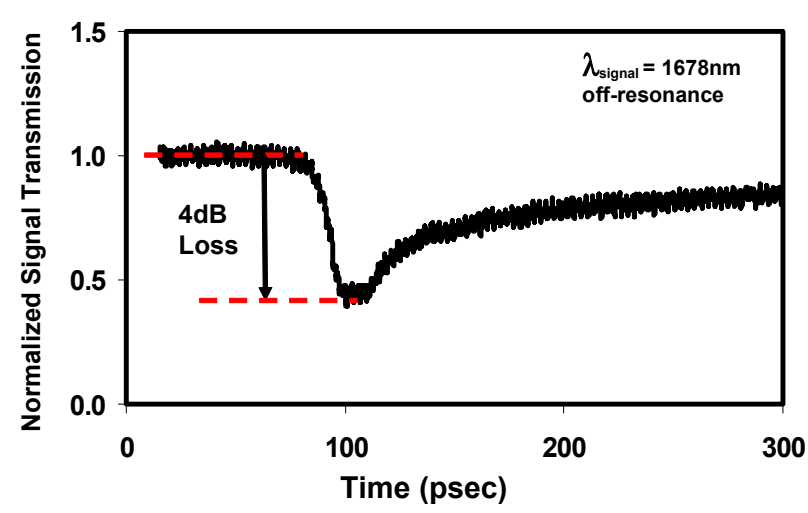

Figure 7: Time resolved signal loss with the signal laser at $1678 \mathrm{~nm}$, ie. outside the Raman resonance. Maximum loss of $4 \mathrm{~dB}$ and carrier lifetime of $4 \mathrm{~ns}$ is obtained.

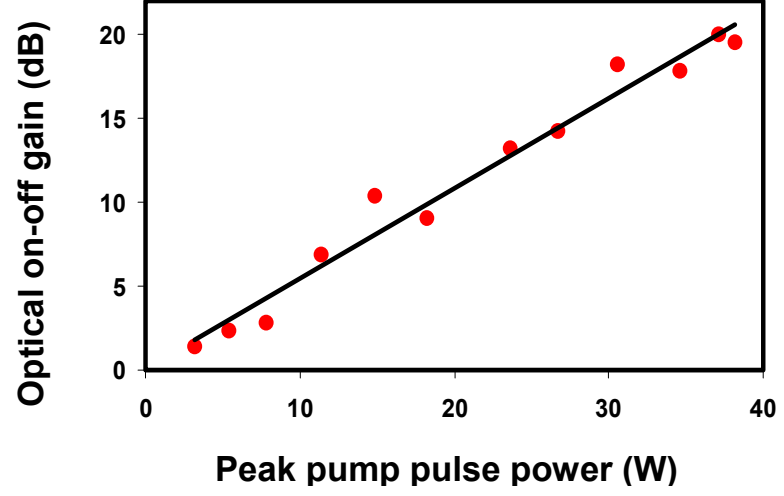

Figure 8: On-off optical gain as a function of peak pump pulse power. Maximum gain of $20 \mathrm{~dB}$ is obtained.

is measured to be $4 \mathrm{~dB}$. Thus the intrinsic Raman gain in the Silicon waveguide is $24 \mathrm{~dB}$. By extrapolating the exponential decay of the carriers we estimate a free carrier lifetime of $\sim 4 \mathrm{nsec}$.

The variation of optical gain as a function of peak pump power coupled into the waveguide is shown in Figure 8. Optical gain is found to saturate around 37W of peak pump power. This can be attributed to the pulse breakup and excessive spectral broadening of pump laser in the fiber pigtail preceding the waveguide [34].

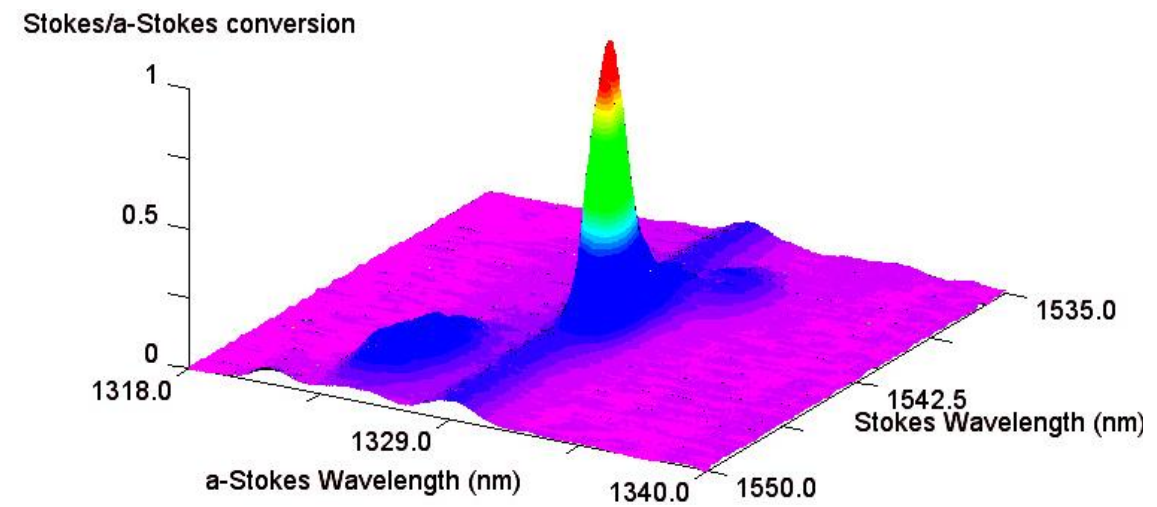

Figure 9. Measured anti-Stokes spectra vs. Stokes signal wavelength. The z-axis represents the conversion efficiency, normalized to unity. C.W. pump laser wavelength is $1428 \mathrm{~nm}$.
Figure 9 shows experimental verficication of Raman wavelength conversion in silicon. The pump laser (at $1428 \mathrm{~nm}$ ) is coupled into the $\mathrm{TE}_{0}$ mode, and the signal laser is coupled into the $\mathrm{TM}_{0}$ mode. The Stokes signal laser is scanned in a range from 1530 to $1560 \mathrm{~nm}$. The Figure 9 shows the a-Stokes spectra measured as a function of the Stokes signal wavelength. The $\mathrm{CW}$ pump power in the waveguide was $0.7 \mathrm{~W}$. There is a clear peak at $1328.8 \mathrm{~nm}$ of anti-Stokes emission when the Stokes laser is tuned to Stokes wavelength of $1542.3 \mathrm{~nm}$. The nature

of the satellite peaks maybe due to the $\sin c^{2}$ dependence of the conversion efficiency with phase mismatch.

Figure 10a shows the converted a-Stokes signal spectrum [39]. The FWHM for wavelength conversion, which is approximately $250 \mathrm{GHz}$, is determined solely by the pump laser linewidth. Figure $10 \mathrm{~b}$ shows the conversion of $1.03 \mathrm{GHz}$ RF modulation from $1542 \mathrm{~nm}$ to $1328.5 \mathrm{~nm}$ [39]. The input RF signal power applied to the Stokes wavelength is shown in the inset. The measured electrical Signal to Noise Ratio (SNR) is $34 \mathrm{dBe}$. We note that from the application point of 
view, the $1320 \mathrm{~nm}$ and $1550 \mathrm{~nm}$ bands are the two most important bands in optical communication. The measured conversion efficiency was approximately $10^{-5}$. As mentioned previously, a number of design approaches are available for
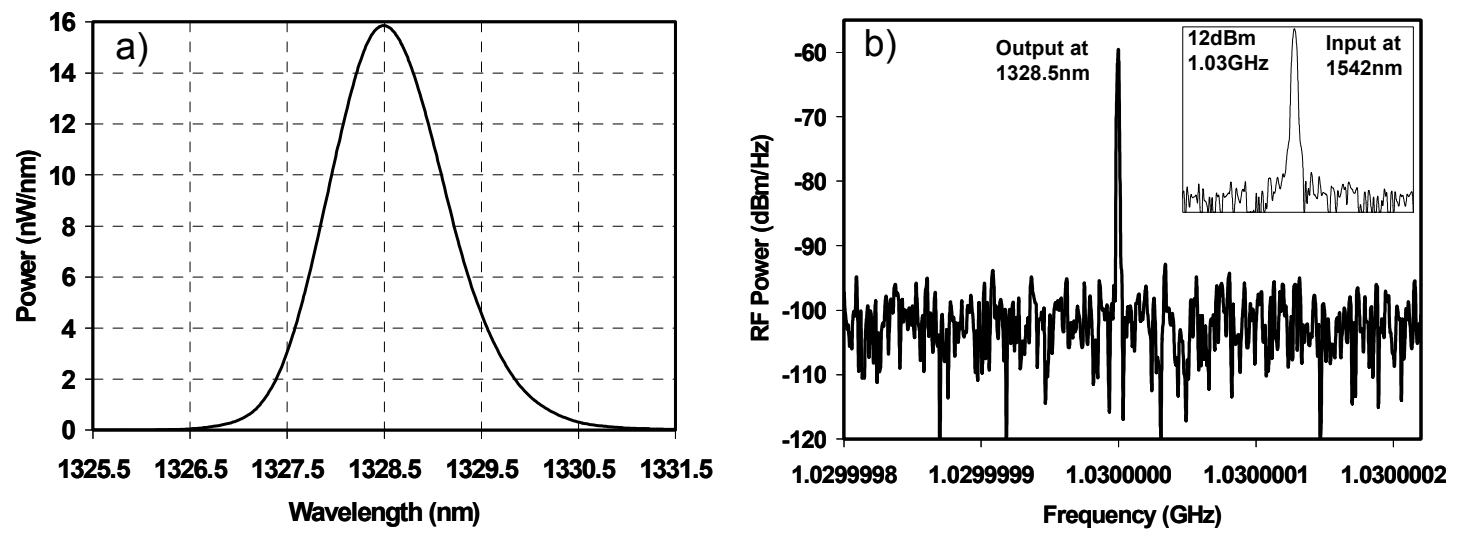

Figure 10. (a) Anti-Stokes spectrum showing a the converted signal (b) RF spectrum at $1328.5 \mathrm{~nm}$ showing conversion of a $1.03 \mathrm{GHz}$ RF modulation from Stokes to anti-Stokes. The inset shows the $R F$ spectrum of the input signal at $1542.3 \mathrm{~nm}$.

phase matching a silicon waveguide and for realizing high conversion efficiency [40].

Figure 11 shows the measure input-output curve for the first silicon Raman laser [4]. The laser, demonstrated in 2004, operated in the pulse mode and consisted of a $1.7 \mathrm{~cm}$ long silicon waveguide gain medium and an external cavity form via a fiber loop. The laser has a threshold of $9 \mathrm{~W}$ peak pulse power (corresponding to a few $\mathrm{mW}$ of average power) and was able to produce output pulses with over $2.5 \mathrm{~W}$ peak power at $25 \mathrm{MHz}$ repetition rate. The demonstration was a major milestone as it clearly showed that silicon can indeed lase. The strong lasing characteristics and high conversion efficiency $(\sim 13 \%)$ of the prototype laser showed that silicon Raman lasers must be considered as a practical and compact

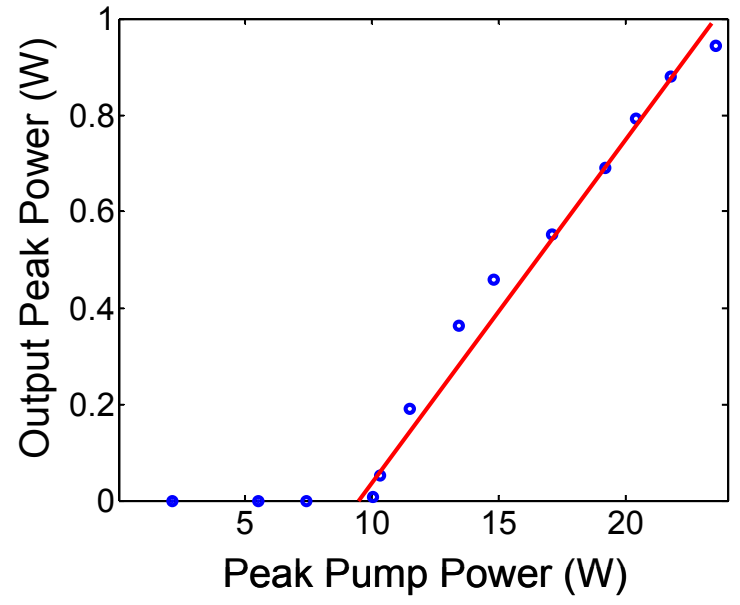

Figure 11. Measured laser output power with respect to peak pump power. Lasing threshold is measured to be at $9 \mathrm{~W}$ peak power level. The slope efficiency obtained by dividing the output peak pulse power by that of the input is $\sim 13 \%$. alternative to fiber Raman lasers.

In 2005, Intel Corporation demonstrated the first CW silicon Raman laser [6]. The device was a $5 \mathrm{~cm}$ long silicon waveguide with a cavity formed by HR coating the chip facets. $\mathrm{CW}$ operation was achieved by using a reverse bias $p-n$ junction to sweep out the TPA generated free carriers, an approach that was previously proposed in 2004 [23-24, 30]. Figure 12 shows the laser input-output behavior at reverse bias voltages of 5 and $25 \mathrm{~V}$. The laser produced a maximum output power of $\sim 9 \mathrm{~mW}$ at $25 \mathrm{~V}$ reverse bias with $600 \mathrm{~mW}$ of $\mathrm{cw}$ pump power inside the waveguide. The cw operation is an important step in the development of silicon Raman lasers. One drawback of the reverse bias carrier sweep out approach is the electrical power dissipated on the chip. With a reverse current of approximately $50 \mathrm{~mA}$ expected for this device, the laser dissipates an on-chip electrical power of more than $1 \mathrm{~W}$. From this perspective, methods that can drastically reduce carrier lifetime, and hence mitigate the need for active carrier removal, are a desired. As a compromise, reduction of

the required sweep out voltage will reduce the electrical power dissipation.

The ability to integrate a p-n junction along with the gain medium offers the exciting possibility of intra-cavity gain switching [5]. By injecting free carriers into the gain medium, cavity loss can be modulated leading the direct electrical 


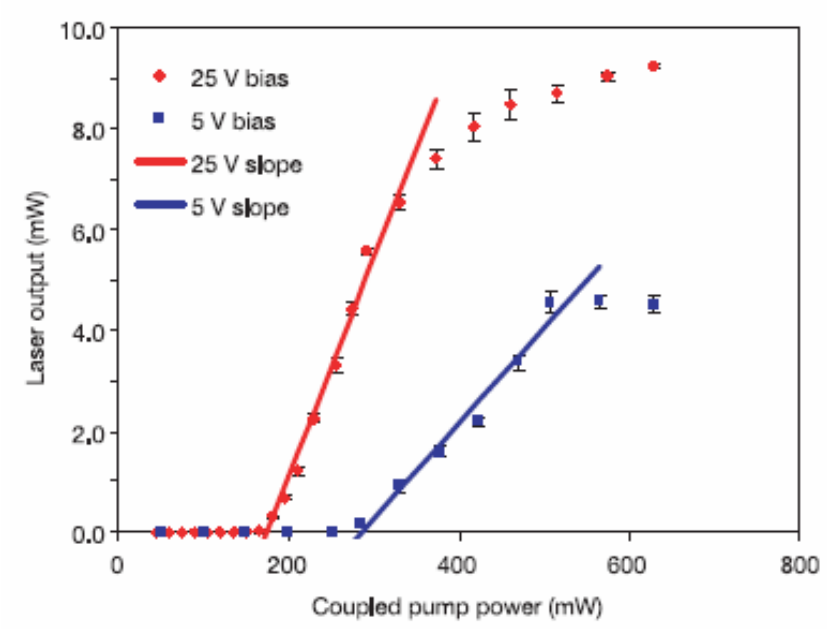

Figure 12. Threshold characteristics of $\mathrm{CW}$ silicon Raman laser demonstrated in a $5 \mathrm{~cm}$ silicon waveguide and with using reverse biased $p$-i-n diode for carrier sweep out [6]. modulation of the laser output. Using this technique, direct laser switching with $30 \mathrm{~dB}$ extinction ratio has been demonstrated [5]. This is a unique feature of silicon Raman lasers that is not possible in fiber Raman lasers. It allows the laser to be interfaced with on-chip electronic circuitry in all-silicon optoelectronic integrated circuits. This idea, first demonstrate by UCLA, has been extended by Intel in a demonstration of an electrically switched Raman amplifier, with the device representing a loss-less modulator [41].

\section{GeSi Raman Devices}

The introduction of germanium in the overall scheme of nonlinear Raman processes in silicon offers new avenues for tailoring the device characteristics. In particular [42]:

(i) The strain caused by the difference in the lattice constants of Si and Ge along with the composition effect, provide mechanisms for tuning the Stokes shift associated with the dominant $\mathrm{Si}-\mathrm{Si}(500 \mathrm{~cm}-1)$

vibrational mode [14]. In addition, the presence of Si-Ge modes $(400 \mathrm{~cm}-1)$ and Ge-Ge modes $(300 \mathrm{~cm}-1)$ provide flexibility in pump and signal wavelengths.

(ii) Spectral broadening can be achieved by via graded Ge composition.

(iii) The strain resulting in birefringence $[15,16]$ can provide an addition degree of freedom for phase matching in the wavelength conversion process. Stress also results in broadening of the gain spectrum, via splitting of the degenerate optical phonon modes.

(iv) When grown on an SOI substrate, the use of double cladding in the vertical direction can improve fiber waveguide coupling efficiency.

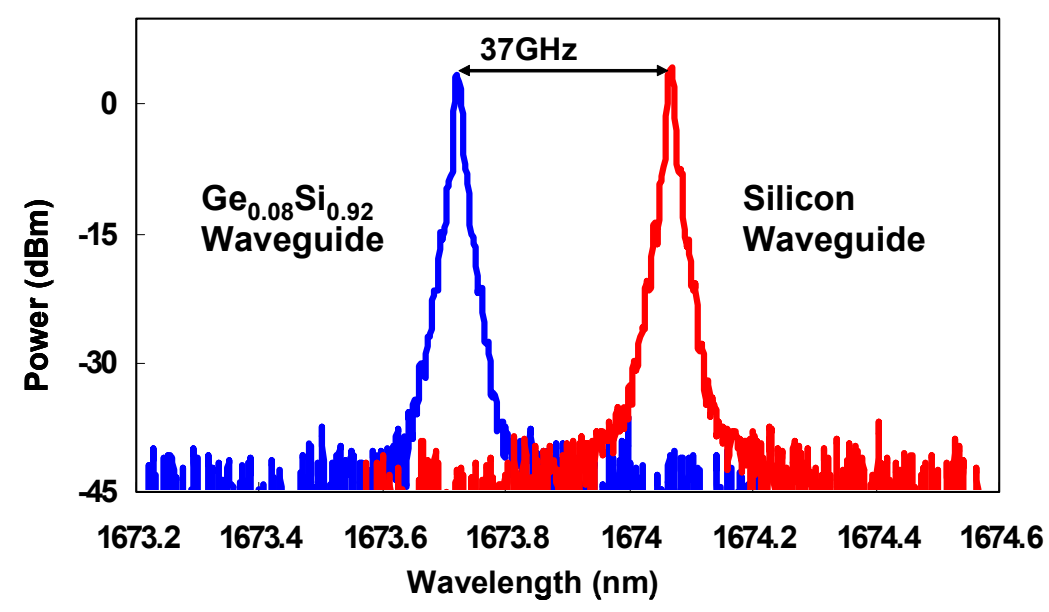

Figure 13 Stimulated Raman spectra of GeSi waveguides compared to that of silicon waveguide. A $37 \mathrm{GHz}$ red shift in the Stokes wavelength is observed. Pump wavelength was 1539nm [42]. 
(v) Higher carrier mobility, and hence diffusion constant, in SiGe reduces the effective lifetime in the waveguide. This reduces the losses associated with the free carriers that are generated by Two Photon Absorption (TPA). However, this benefit will be countered by the higher TPA coefficient in GeSi.

Recently, the first GeSi optical amplifier and laser was demonstrated [42]. A pulsed gain of 14dB and lasing with sharp threshold characteristics were observed for $\mathrm{Ge}_{0.08} \mathrm{Si}_{0.92}$ rib waveguides. The Stokes spectrum, shown in Figure 13, exhibits a $37 \mathrm{GHz}$ red shift which is in qualitative agreement with a model that takes into account the effect of composition and strain on the optical phonon frequency [38]. These results suggest that the spectrum of Raman scattering can be engineered using the GeSi material system. As a result, GeSi Raman devices represent an exciting topic for future research and development.

\section{Summary}

This manuscript has outlined light generation, amplification and wavelength conversion in silicon and GeSi using stimulated Raman processes. These effects are routinely observed in optical fiber; however, several kilometers of length are required to do so. What has enabled us to achieve these processes on millimeter length scales on a chip are two fundamental differences between an optical fiber and a silicon microstructure. The first is the difference in atomic structures. Vibrational modes in silica broaden into bands; hence the Raman gain has a very large bandwidth and a low peak value, requiring long interaction lengths for the effect to be observed. In contrast, silicon is single crystal and supports only 3 degenerate optical vibration modes. The result is a much narrower gain bandwidth but a much higher gain peak (nearly $10^{3}-10^{4}$ times higher). Second, is the difference in modal areas. The large index-contrast in the silicon $/ \mathrm{SiO}_{2}$ waveguides results in a mode area that is approximately 100 times smaller (assuming $0.8 \mu \mathrm{m}^{2}$ waveguides) than in a standard single mode fiber $\left(\right.$ mode area $\left.=80 \mu \mathrm{m}^{2}\right)$. The proportionally higher power density in the silicon waveguide lowers the threshold for nonlinear optical processes.

The intrinsic Raman bandwidth in silicon is $105 \mathrm{GHz}$, and it is broadened in the experiments by the pump laser linewidth (typically $\sim 2 \mathrm{~nm}$ ). The resulting bandwidth is sufficient for amplifying several WDM channels. The bandwidth can be broadened by broadening the pump linewidth even further (although this reduces the peak gain) or by using multiple pump wavelengths. The Raman phenomenon is fully tunable; the tuning range is only limited by the available pump wavelengths. This is an advantage over the nanocrystal approach for light generation and amplification. In addition, the Raman Effect can also perform wavelength conversion. Another important advantage of this approach is that it does not require rare-earth dopants or nanostructures. Hence, it is truly compatible with silicon IC manufacturing. A limitation of the Raman approach is the fact that it is optically pumped. However, it has been shown that Silicon Raman lasers can be electronically switched or modulated using intra-cavity gain-loss modulation [6]. Therefore, these lasers or similarly amplifiers can be interfaced with on-chip electronic circuitry. Raman amplification and lasing in GeSi waveguides has recently been demonstrated. The GeSi material system provides an opportunity to engineer the, otherwise rigid, Raman spectrum of silicon. Owing to the enhance carrier mobility, GeSi is being pursued by the CMOS IC industry for future high speed circuits. This provides another impetus for investigating GeSi Raman devices.

Going forward, low loss waveguides with small cross sections are required for realizing high performance devices. Surface roughness produces strong scattering and high propagation loss due to the high index contrast between the silicon waveguide core and the cladding (air or $\mathrm{SiO}_{2}$ ). As such, the losses of silicon waveguides tend to increase with reduction in cross section. Fortunately, new waveguide fabrication processes that are in development promise low-loss waveguides with submicron cross sections [43-44]. Naturally, one must be able to efficiently couple light into these structures. Several novel approaches for high efficiency coupling into submicron waveguides have also been developed [44].

\section{References}

[1] See for example: www.kotura.com

[2] M. Paniccia, M. Morse, M. Salib; “Integrated Photonics”, ch. 2 in L. Pavesi, D.J. Lockwood (Eds.), 
Silicon Photonics, Topics in Appl. Phys. 94, 51-88 (2004).

[3] Z. Gaburro; "Optical Interconnect", ch. 4 in L. Pavesi, D.J. Lockwood (Eds.), Silicon Photonics, Topics in Appl. Phys. 94, 121-176 (2004).

[4] Ozdal Boyraz and Bahram Jalali, "Demonstration of a silicon Raman laser," Opt. Express 12, 5269-5273 (2004),

[5] O. Boyraz and B. Jalali, "Demonstration of directly modulated silicon Raman laser," Opt. Express 13, 796-800 (2005).

[6] Haisheng Rong, Richard Jones, Ansheng Liu1, Oded Cohen, Dani Hak, Alexander Fang and Mario Pannicia, A continuous-wave Raman silicon laser"Nature 433, 725 - 728, 2005

[7] B. Jalali, S. Yegnanarayanan, T. Yoon, T. Yoshimoto, I. Rendina, F. Coppinger, "Advances in Silicon-on-Insulator Optoelectronics", IEEE J. of Sel. Top. in Quant. El. 4, 938 - 947 (1998).

[8] R. Claps, D. Dimitropoulos, Y. Han, B. Jalali, "Observation of Raman emission in silicon waveguides at $1.54 \mu \mathrm{m}$," Opt. Exp. 10, 1305-1313 (2002).

[9] R. Claps, D. Dimitropoulos, V. Raghunathan, Y. Han, B. Jalali, "Observation of stimulated Raman scattering in Silicon waveguides", Opt. Exp. 11, 1731-1739 (2003).

[10] R. Claps, V. Raghunathan, D. Dimitropoulos, B. Jalali, “Anti-stokes Raman conversion in silicon waveguides”, Opt. Exp. 11, 2862-2872 (2003).

[11] A. Yariv, Quantum Electronics, $3^{\text {rd }}$ ed., John Wiley and Sons, New York (1988). ISBN 0-471-60997-8.

[12] Peter Y. Yu and Manuel Cardona, Fundamentals of Semiconductors, $3^{\text {rd }}$ Edition, Springer.

[13] P.A. Temple, C.E. Hathaway, "Multiphonon Raman Spectrum of Silicon”, Phys. Rev. A 7, 3685 (1973).

[14] D. Dimitropoulos, B. Houshmand, R. Claps, B. Jalali, "Coupled-mode theory of Raman effect in silicon-oninsulator waveguides", Opt. Lett. 28 (2003).

[15] J.M. Ralston, R.K. Chang, "Spontaneous-Raman-scattering efficiency and stimulated scattering in silicon", Phys. Rev. B 2, 1858-1862 (1970).

[16] J.H. Yee, H.H.M. Chau; “Two-Photon indirect transition in GaP crystal,” Opt. Comm. 10, 56-58 (1974).

[17] K.W. DeLong, G.I. Stegeman; "Two-photon absorption as a limitation to all-optical waveguide switching in semiconductors,” Appl. Phys. Lett. 57(20) 2063-2064 (1990).

[18] A. Villeneuve, C.C. Yang, G.I. Stegeman, C.N. Ironside, G. Scelsi, R.M. Osgood; "Nonlinear Absorption in a GaAs Waveguide Just Above Half the Band Gap," IEEE J. Quant. Electron. 30, 1172-1175 (1994).

[19] A.M. Darwish, E.P. Ippen, H.Q. Lee, J.P. Donnelly, S.H. Groves; "Optimization of four-wave mixing conversion efficiency in the presence of nonlinear loss," Appl. Phys. Lett. 69, 737-739 (1996).

[20] Y.-H. Kao, T.J. Xia, M.N. Islam; "Limitations on ultrafast optical switching in a semiconductor laser amplifier operating at transparency current", J. Appl. Phys. 86, 4740-4747 (1999).

[21] K. Suto, T. Kimura, T. Saito, J. Nishizawa; "Raman amplification in GaP-AlxGa1-xP waveguides for light frequency discrimination,” IEE Proc.-Optoelectron. 145, 105-108 (1998).

[22] S. Saito, K. Suto, T. Kimura, J.I. Nishizawa; "80-ps and 4-ns Pulse-Pumped Gains in a GaP-AlGaP Semiconductor Raman Amplifier," IEEE Photon. Technol. Lett.16, 395-397 (2004).

[23] T.K. Liang, H.K. Tsang; "Role of free carriers from two-photon absorption in Raman amplification in silicon-oninsulator waveguides," Appl. Phys. Lett. 84(15) 2745-2747 (2004).

[24] R. Claps, V. Raghunathan, D. Dimitropoulos, and B. Jalali, "Influence of nonlinear absorption on Raman amplification in Silicon waveguides," Opt. Express 12, 2774-2780 (2004),

[25] A. R. Cowan, G. W. Rieger, and J. F. Young, "Nonlinear transmission of $1.5 \mu \mathrm{m}$ pulses through single-mode silicon-on-insulator waveguide structures," Opt. Express 12, 1611-1621 (2004), 
[26]R. A. Soref, B. R. Bennett; “Electrooptical Effects in Silicon,” IEEE J. Quant. Electron. QE-23, 123-129 (1987).

[27]R. J. Bozeat, S. Day, F. Hopper, F.P. Payne, S.W. Roberts, M. Asghari, "Silicon Based Waveguides," in L. Pavesi, D.J. Lockwood (Eds.) Silicon Photonics, ch. 8, 269-294 (2004).

[28] M.A. Mendicino; "Comparison of properties of available SOI materials," Properties of Crystalline Silicon, by Robert Hull 18.1 p. 992-1001 (1998).

[29] J.L. Freeouf, S.T. Liu; IEEE Int. SOI conf. proc. Tucson, AZ, USA, 3-5 Oct, 1995 p. 74-5.

[30] D. Dimitropoulos, R. Jhaveri, R. Claps, J. C. S. Woo, and B. Jalali Lifetime of photogenerated carriers in silicon-oninsulator rib waveguides, Appl. Phys. Lett. 86, 071115 (2005)

[31] R. Espinola, J. Dadap, R. Osgood, S.J. McNab, Y. A. Vlasov; "Raman amplification in ultrasmall silicon-oninsulator wire waveguides", Opt. Exp. 12(16), 3713-3718 (2004).

[32] R. Jones, H. Rong, A. Liu, A. W. Fang, M. J. Paniccia, D. Hak, and O. Cohen, "Net continuous wave optical gain in a low loss silicon-on-insulator waveguide by stimulated Raman scattering," Opt. Express 13, 519-525 (2005)

[33] E. Golovchenko, P.V. Mamyshev, A.N. Pilipetskii, E.M.Dianov, "Mutual influence of the Parametric effects and Stimulated Raman scattering in Optical fibers", IEEE. J. Quant. Elec., 26, 1815-1820 (1990).

[34] Ozdal Boyraz and Bahram Jalali, "Demonstration of $11 \mathrm{~dB}$ fiber-to-fiber gain in a silicon Raman amplifier", IEICE Electron. Express, Vol. 1, No. 14, pp.429-434, (2004)

[35] T. K. Liang and H.K. Tsang, "Efficient Raman amplification in silicon-on-insulator waveguides", Appl. Phys. Lett. $85,3343-3345$ (2004).

[36] Q. Xu, V.R. Almeida and Michal Lipson, "Time-resolved study of Raman gain in highly confined silicon-oninsulator waveguides", Opt. Exp. 12, 4437-4442 (2004).

[37] A. Liu, H. Rong, M. Paniccia, O. Cohen, and D. Hak, "Net optical gain in a low loss silicon-on-insulator waveguide by stimulated Raman scattering, " Optics Express, vol. 12, no. 18, pp. 4261-4268, Sept. 2004.

[38] Varun Raghunathan, Ozdal Boyraz and Bahram Jalali, "20 dB on-off Raman amplification in silicon waveguides," CLEO 2005, Baltimore, MD, May 2005, CMU1.

[39] V. Raghunathan, R. Claps, D. Dimitropoulos and B. Jalali, "Wavelength Conversion in Silicon Waveguides using Raman-induced four wave mixing", Appl. Phys. Lett. 85(1), 34-36 (2004).

[40] D. Dimitropoulos, V. Raghunathan, R. Claps, B. Jalali, "Phase-matching and Nonlinear Optical Processes in Silicon Waveguides", Optics Exp. 12, 2774 (2003).

[41] Richard Jones, Ansheng Liu, Haisheng Rong, Mario Paniccia, Oded Cohen, and Dani Hak, "Lossless optical modulation in a silicon waveguide using stimulated Raman scattering", Optics Express, Vol. 13, No. 5, March 07, 2005, pp. $1716-1723$

[42] Ricardo Claps, Varun Raghunathan, Ozdal Boyraz, Prakash Koonath, Dimitrios Dimitropoulos, and Bahram Jalali "Raman amplification and lasing in SiGe waveguides," Optics Express, Vol. 13, No. 7, 4 April 2005, p. 2459

[43] P. Koonath, K. Kishima, T. Indukuri, and B. Jalali, "SIMOX sculpting of 3-D nano-optical structures," LEOS Annual Meeting, Tucson, AZ, (October 2003).

[44] Tai Tsuchizawa, Koji Yamada, Hiroshi Fukuda, Toshifumi Watanabe, Jun-ichi Takahashi, Mitsutoshi Takahashi, Tetsufumi Shoji, Emi Tamechika, Sei-ichi Itabashi, and Hirofumi Morita, "Microphotonics Devices Based on Silicon Microfabrication Technology” IEEE Journal of Selected Topics in Quantum Electronics, Vol. 11, No. 1, January/February 2005, pp. 232-240. 